\title{
Elaboração de uma Matriz de Avaliação e Monitoramento de uma Farmácia Hospitalar
}

\author{
Lucélia Hernandes Lima ${ }^{1}$, Carine Raquel Blatt ${ }^{2}$, \\ Marcelo Schenk de Azambuja²
}

\begin{abstract}
RESUMO
Introdução: O acompanhamento e a avaliação continuada das ações desenvolvidas na Assistência Farmacêutica são estratégias para a busca da qualidade, especialmente no âmbito hospitalar. Uma boa avaliação visa a reduzir incertezas, melhorar a efetividade e monitoramento das ações e propiciar as tomadas de decisão relevantes, auxiliando na gestão dos serviços. Objetivos: Elaborar uma matriz de avaliação e monitoramento de uma farmácia hospitalar. Método: Trata-se de um estudo avaliativo. Para a elaboração e validação de conteúdo foram desenvolvidas as seguintes etapas: revisão bibliográfica, proposição das dimensões e elaboração de indicadores, grupo focal com especialistas para validação e priorização das dimensões e aplicação da técnica delphi para relevância e valoração dos indicadores. O coeficiente alfa de Cronbach foi utilizado para medir a confiabilidade dos indicadores. O estudo foi desenvolvido no contexto da farmácia de um hospital de grande porte no Estado do Rio Grande do Sul. Resultados: Foram identificadas 5 dimensões para a avaliação da Farmácia Hospitalar e 70 indicadores, distribuídos da seguinte maneira: Gestão da Assistência ( $n=15$ ), Gestão dos Recursos Humanos ( $n=13)$, Gestão do Medicamento ( $n=13)$, Gestão Financeira ( $n=12$ ) e Gestão da Clínica dos Pacientes ( $n=17)$. A dimensão Gestão do Medicamento foi priorizada pelos especialistas para validação e valoração. Essa dimensão resultou em uma matriz, com 9 indicadores. Conclusão: $O$ estudo evidenciou a importância da avaliação e monitoramento para a gestão da Farmácia Hospitalar e a capacidade de execução de métodos de consenso para indicadores de qualidade em serviços de alto nível de complexidade. O instrumento com os indicadores desenvolvidos para dimensão Gestão do Medicamento mostrou evidência de validade e uma boa confiabilidade.
\end{abstract}

Palavras-chave: Avaliação de processos; resultados em cuidados de saúde; farmácia hospitalar; indicadores de gestão; gestão da qualidade total.

\section{FORMULATE OF A FRAMEWORK TO EVALUATED AND MONITORING OF A HOSPITAL PHARMACY SERVICES}

\section{ABSTRACT}

Introduction: The monitoring and continuous evaluation of the actions developed in Pharmaceutical Care are strategies for the search for quality, especially in hospitals. A good evaluation aims to reduce uncertainties, improve the effectiveness and monitoring of actions and provide relevant decision-making by assisting in the service management policy. Objective: To develop an evaluation and monitoring matrix for a pharmacy in a hospital. Method: It is an evaluative study. In order to elaborate and validate the content the following steps have been developed: bibliographic review, proposition on dimensions and elaboration of indicators, focus group with experts to validate and prioritize the dimensions and application of the delphi technique to the indicators for relevance and valuation. Cronbach's alpha coefficient has been used to measure the reliability of the indicators. Results: Five dimensions and 70 indicators have been identified for the evaluation of Hospital Pharmacy, distributed as follows: Care Management $(n=15)$, Human Resources Management $(n=13)$, Drug Management $(n=13)$, Financial Management $(n=12)$ and Patient Clinic Management $(n=17)$. The dimension Drug Management has been prioritized by the specialists for validation and valuation. This dimension resulted in a matrix, with nine indicators. Conclusion: The study has shown the importance of evaluation and monitoring for Hospital Pharmacy management and the capacity of executing consensus methods to quality indicators in high complexity services. The instruments with indicators developed for the dimension Drug Management has shown evidence of validity and good reliability.

Keywords: Process assessment; health care results; hospital pharmacy; management indicators; total quality management.

RECEBIDO EM: 9/11/2020

MODIFICAÇÕES SOLICITADAS EM: 20/5/2021

ACEITO EM: 24/6/2021

\footnotetext{
${ }^{1}$ Autora correspondente. Irmandade da Santa Casa de Misericórdia de Porto Alegre. Rua Professor Annes Dias, 295 - Centro Histórico. Porto Alegre/RS, Brasil. CEP 90020-090. http://lattes.cnpq.br/3117391621425144. https://orcid.org/0000-00016595-5623. luceliahernandes@gmail.com

2 Universidade Federal de Ciências da Saúde de Porto Alegre (UFCSPA). Porto Alegre/RS, Brasil.
} 


\section{INTRODUÇÃO}

Conceitualmente, Farmácia Hospitalar (FH) é uma unidade clínico-assistencial, técnica e administrativa, na qual se processam as atividades relacionadas à Assistência Farmacêutica (AF), dirigida exclusivamente por farmacêuticos, compondo a estrutura organizacional do hospital e integrada funcionalmente com as demais unidades administrativas e de assistência ao paciente. ${ }^{1}$ Seu principal objetivo é contribuir no processo de cuidado à saúde, visando a melhorar a qualidade da assistência, promovendo o uso seguro e racional de medicamentos e produtos para a saúde. ${ }^{2}$

As atividades básicas da FH estão relacionadas com a seleção, logística, informação e gerenciamento de medicamentos e produtos médicos hospitalares. ${ }^{3}$ O aumento da complexidade das atividades desenvolvidas no hospital agrega uma série de outras atividades a serem desenvolvidas pelo Serviço de Farmácia, para que este se torne compatível com as necessidades da instituição. Sendo assim, os serviços de farmácia apresentam características diferentes de acordo com o hospital no qual estão inseridos. ${ }^{4}$

A avaliação é um dos mecanismos de controle de qualidade dos serviços de saúde. Segundo Donabedian, ${ }^{5}$ principal autor que se dedicou a estudar e publicar sobre o processo da qualidade na área da saúde, avaliar é monitorar continuamente os serviços de saúde oferecidos, para detectar e corrigir precocemente os desvios dos padrões encontrados, permitindo o aperfeiçoamento e desenvolvimento dos serviços avaliados. ${ }^{6}$

Qualidade pode ser abordada tanto de forma abrangente quanto de forma delimitada, pois assume diferentes significados: qualidade de trabalho, serviço, informação, processo, estrutura e pessoas. Ela implica a satisfação das expectativas e necessidades dos clientes por meio de uma gestão científica dos processos, baseada em fatos e dados, voltada para a correção e prevenção de erros. ${ }^{7}$ Qualidade em saúde pode ser definida como "o grau em que os serviços de saúde, para indivíduos e populações, aumentam a probabilidade de desfechos de saúde desejados e são consistentes com o conhecimento profissional atual". 8

Os desfechos de saúde desejados relacionam-se com os objetivos dos serviços hospitalares, que se fundamentam na prestação da assistência curativa e preventiva à população. Entende-se que esses objetivos serão alcançados no atendimento das necessidades do paciente, na prestação de um cuidado livre de dano e diante da satisfação desse paciente. A prestação de serviços de saúde, no ambiente hospitalar, é um processo dinâmico que envolve muitas etapas integradas e interdependentes. Cada uma dessas etapas tem o potencial de falhar. A falha em qualquer etapa pode resultar em um risco de dano ao paciente e aumentar a morbidade e mortalidade ${ }^{9}$.

A segurança do paciente está relacionada à redução a um mínimo aceitável de risco de dano desnecessário associado ao cuidado de saúde. ${ }^{2}$ As organizações de saúde estão buscando, em métodos de melhoria da qualidade, estratégias para garantir processos mais estruturados, com práticas definidas e disseminadas na instituição e, assim, assegurar a esses clientes melhores assistência e segurança.

Editora Unijuí - Revista Contexto \& Saúde - ISSN 2176-7114 - v. 21, n. 44, out./dez. 2021 
A gestão da qualidade surge como ferramenta de apoio que auxilia na reestruturação dos processos internos, gerando benefícios para os usuários e para os colaboradores, tanto na configuração das atividades de trabalho como na manutenção do foco na infraestrutura. ${ }^{7}$ Dessa forma, se a melhoria da qualidade está relacionada à busca por incrementar a segurança do paciente, o termo gestão busca interiorizar e envolver outros níveis da governança.

Em um hospital, quanto maior a sua habilidade de organização e de sua farmácia em organizar os seus processos, maior será sua capacidade de oferecer aos clientes serviços de qualidade e com baixos custos operacionais. Dessa maneira, este estudo tem como objetivo elaborar uma matriz de indicadores para a avaliação e monitoramento da farmácia de uma instituição hospitalar.

\section{METODOLOGIA}

Trata-se de um estudo avaliativo de elaboração de indicadores de avaliação e monitoramento da farmácia hospitalar. Para a elaboração e validação de conteúdo do instrumento avaliativo, foram desenvolvidas as seguintes etapas: revisão bibliográfica, proposição das dimensões e elaboração de indicadores, grupo focal com especialistas para validação e priorização das dimensões e aplicação da técnica delphi para validação e valoração dos indicadores.

$\mathrm{Na}$ etapa revisão bibliográfica foi realizada uma busca por artigos que apresentassem indicadores para a avaliação da Farmácia Hospitalar ou resultados de sua avaliação. A busca foi realizada a partir dos descritores key performance indicator e pharmacy hospital nas bases de dados National Library of Medicine (PubMed) e SciELO Library.

Os critérios de inclusão compreenderam artigos que contemplavam a elaboração e validação de conteúdo de modelos de avaliação de serviços farmacêuticos hospitalares, com resumos e artigo completo disponíveis eletronicamente nos idiomas português, inglês e espanhol e com uma limitação temporal de publicação entre 2014 e julho de 2019. Foram selecionados os tópicos melhoria da qualidade, farmácia e hospital para refinar a busca. Foram excluídos da revisão os artigos que não disponibilizarem o resumo na base de indexação e os estudos duplicados.

Para a proposição das dimensões foi utilizado o mapeamento de processos do Serviço de Farmácia desenvolvido na instituição pesquisada.

A elaboração dos indicadores baseou-se nas dimensões propostas e seus atributos, definidos com base no mapeamento de processos da instituição investigada. Para cada dimensão foram identificados dados e fontes de dados que pudessem representar uma etapa do processo na respectiva dimensão. A partir desse ponto buscou-se na literatura indicadores previamente utilizados por diferentes serviços. $\mathrm{Na}$ ausência de indicadores preestabelecidos foi realizada a proposição de novos indicadores. Na construção, foram desenvolvidas as fichas técnicas dos indicadores. Elas são constituídas dos elementos considerados lógicos para a composição dos indicadores: nome, objetivo, método de cálculo e frequência de aferição.

Editora Unijuí - Revista Contexto \& Saúde - ISSN 2176-7114 - v. 21, n. 44, out./dez. 2021 
Para a validação e priorização das dimensões e dos indicadores propostos foi convidado um grupo de especialistas. Na seleção, foram considerados aptos todos os profissionais farmacêuticos da instituição investigada, desconsiderando se a área de atuação era o local de estudo.

A escolha da técnica de grupo focal para esta etapa da pesquisa baseou-se no seu objetivo principal, que é revelar as percepções dos participantes sobre um tema específico. Ela representa uma técnica de coleta de dados por meio das interações entre os participantes a respeito de tópicos pautados em uma discussão. ${ }^{10} \mathrm{O}$ grupo focal teve como objetivo avaliar o grau de relevância dos processos mapeados, distribuídos por dimensões. O resultado dessa avaliação definiu a dimensão prioritária que foi discutida nas próximas etapas.

Para a dimensão selecionada foram discutidos os indicadores, e o grupo de especialistas realizou a validação de conteúdo e valoração dos indicadores.

Foram utilizadas as seguintes perguntas norteadoras para avaliação do conteúdo dos indicadores: "Este indicador foi redigido de forma que o conceito esteja compreensível e expressa adequadamente o que se espera medir?" e "Os elementos de composição do indicador realmente refletem os conceitos envolvidos, são relevantes e adequados para atingir os objetivos propostos?" A cada questionamento pronunciado as modificações foram sendo sugeridas e discutidas pelo grupo.

A segunda parte da aplicação do painel de especialistas teve como objetivo a atribuição de valor aos indicadores realizada por eles. A nota final da dimensão é 10, e foi atribuído um peso específico a cada indicador. As metas foram estabelecidas considerando o referencial teórico e, para os indicadores em uso na instituição, utilizou-se resultados de série histórica.

A validade de conteúdo é uma avaliação qualitativa muito utilizada nos processos de construção de novos instrumentos de medida. Ela consiste no julgamento subjetivo por um comitê de especialistas, que avaliam a qualidade do instrumento, sua clareza, pertinência, relevância e representatividade dos itens, e se cada domínio foi adequadamente abordado pelo conjunto de itens. ${ }^{11}$

Confiabilidade é a capacidade em reproduzir um resultado de forma consistente no tempo e no espaço, ou a partir de observadores diferentes. ${ }^{12-14}$ Para a avaliação da confiabilidade foi realizado o teste de consistência interna, por meio do coeficiente alfa de Cronbach. Esse coeficiente revela a homogeneidade dos itens de uma escala. ${ }^{11}$ Os valores de alfa variam de 0 a 1,0, e quanto mais próximo de 1, maior confiabilidade entre os indicadores. Em termos gerais, o coeficiente alfa de Cronbach mede a correlação entre as respostas em um questionário mediante a análise do perfil das respostas dadas pelos respondentes. ${ }^{15}$

Para a interpretação do coeficiente de alfa de Cronbach foi adotado coeficiente acima de 0,70 , para que se considere satisfatório um instrumento de pesquisa. ${ }^{11,12,16}$

O projeto foi submetido e aprovado pela Comissão de Ética da Irmandade Santa Casa de Misericórdia de Porto Alegre e, como coparticipante, pelo Comitê de Ética da Universidade Federal de Ciências da Saúde de Porto Alegre, parecer consubstanciado número 2.739.632. 


\section{RESULTADOS}

A revisão bibliográfica resultou em 371 artigos. Após a leitura dos títulos e do resumo, apenas três artigos foram selecionados, ${ }^{17-19}$ conforme pode ser visualizado na Figura 1.

Figura 1 - Fluxograma de resultado da revisão bibliográfica dos artigos de avaliação da Farmácia Hospitalar

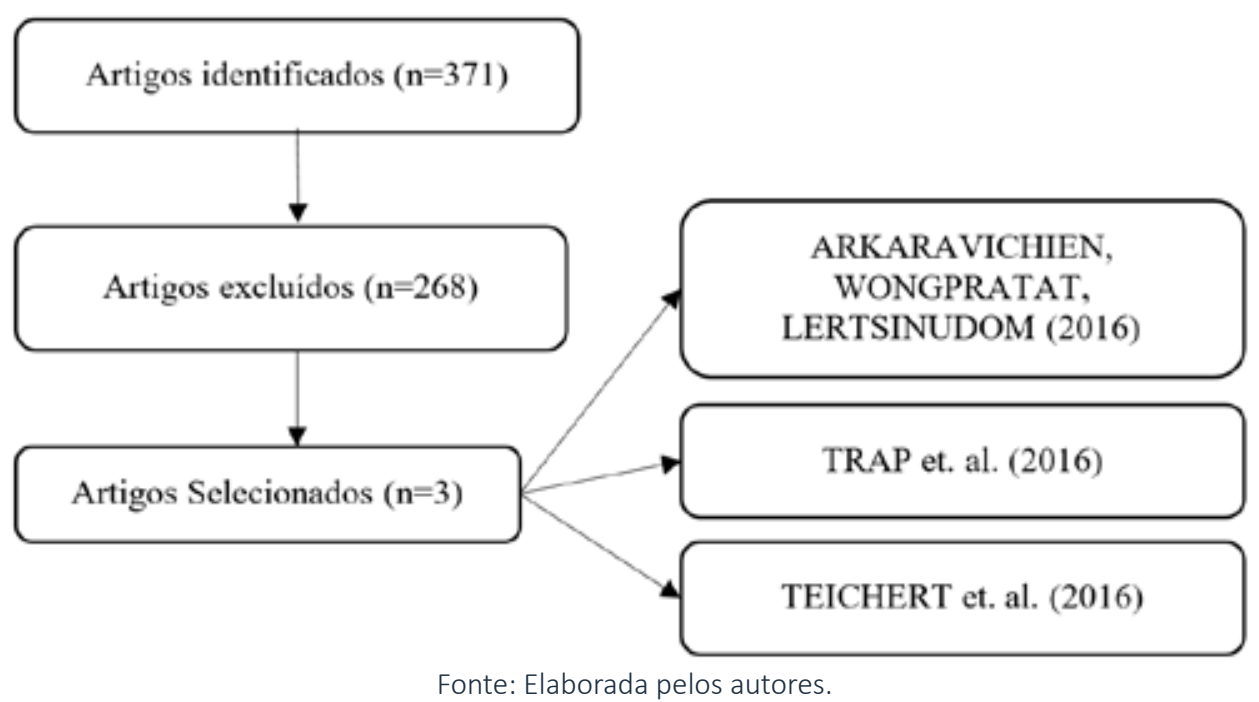

Um estudo transversal em 34 farmácias, composto por 40 itens abrangendo cinco domínios de indicadores de qualidade, foi realizado para a avaliação e melhoria da qualidade das farmácias comunitárias na Tailândia. ${ }^{17}$ Disposições semelhantes foram apresentadas no estudo-piloto realizado pelo Ministério da Saúde de Uganda. ${ }^{18}$ Foram utilizados 25 indicadores classificados em 5 domínios e a cada um deles foi atribuída uma pontuação máxima de 5 , resultando em uma pontuação total máxima na matriz de 25 pontos. O terceiro estudo ${ }^{19}$ teve por objetivo descrever o conjunto de indicadores de qualidade validado para farmácias comunitárias holandesas cujo instrumento era composto de 66 indicadores distribuídos em 10 categorias.

A leitura dos artigos embasou a descrição e caracterização das dimensões propostas para a avaliação. A partir das 5 dimensões propostas, conforme pode ser visualizado na Figura 2, foram elaborados 70 indicadores, distribuídos da seguinte maneira: Gestão da Assistência ( $\mathrm{n}=15)$, Gestão dos Recursos Humanos $(n=13)$, Gestão do Medicamento ( $n=13)$, Gestão Financeira $(n=12)$ e Gestão da Clínica dos Pacientes $(\mathrm{n}=17)$. 
Figura 2 - Dimensões e subdimensões propostas para a avaliação e monitoramento da Farmácia Hospitalar



Fonte: Elaborado pelos autores.

Seis dos 12 farmacêuticos convidados compareceram à reunião do grupo focal. O resultado da relevância das dimensões pelos especialistas destacou as dimensões de Gestão da Assistência e Gestão do Medicamento como as de maior relevância. Uma segunda rodada foi realizada, e a Gestão do Medicamento foi a dimensão priorizada. Dessa maneira, os indicadores propostos para a avaliação desta dimensão foram apresentados e discutidos pelo grupo.

Após essa rodada de discussão, a nova versão dos indicadores, contendo 10 indicadores, foi enviada aos especialistas via correio eletrônico para avaliação da concordância e emissão de juízo de valor. O Quadro 1 apresenta o resultado final dos indicadores para avaliação e monitoramento da dimensão Gestão do Medicamento na Farmácia Hospitalar.

Quadro 1 - Matriz de indicadores para avaliação e monitoramento da dimensão Gestão do Medicamento na Farmácia Hospitalar

\begin{tabular}{|c|c|c|c|c|c|}
\hline Subdimensão & Número & $\begin{array}{l}\text { Nome do } \\
\text { indicador }\end{array}$ & $\begin{array}{l}\text { O que o indicador } \\
\text { mede (informa)? }\end{array}$ & $\begin{array}{l}\text { Como o indicador é } \\
\text { calculado? }\end{array}$ & $\begin{array}{l}\text { Com que } \\
\text { frequência } \\
\text { é medido? }\end{array}$ \\
\hline $\begin{array}{l}\text { Abastecimen- } \\
\text { to }\end{array}$ & 1 & $\begin{array}{l}\text { Atendimento } \\
\text { de abasteci- } \\
\text { mento }\end{array}$ & $\begin{array}{l}\text { Informa o percen- } \\
\text { tual de itens rece- } \\
\text { bidos em relação } \\
\text { aos itens requisita- } \\
\text { dos por dia }\end{array}$ & $\begin{array}{c}\text { (№ total de itens } \\
\text { atendidos / no total } \\
\text { de itens requisitados } \\
\text { por farmácia por dia) } \\
\text { x } 100\end{array}$ & Diário \\
\hline \multirow{2}{*}{$\begin{array}{l}\text { Monitora- } \\
\text { mento dos } \\
\text { Estoques }\end{array}$} & 2 & $\begin{array}{l}\text { Acuracidade } \\
\text { dos estoques }\end{array}$ & $\begin{array}{l}\text { Informa o percen- } \\
\text { tual de acuracida- } \\
\text { de dos itens por } \\
\text { farmácia por mês }\end{array}$ & $\begin{array}{c}\text { (№ de itens confor- } \\
\text { me/no de itens conta- } \\
\text { dos por farmácia por } \\
\text { mês) } \times 100\end{array}$ & Mensal \\
\hline & 3 & $\begin{array}{c}\text { Dias de esto- } \\
\text { que }\end{array}$ & $\begin{array}{c}\text { Informa a quan- } \\
\text { tidade de dias de } \\
\text { estoque disponível } \\
\text { por farmácia por } \\
\text { mês }\end{array}$ & $\begin{array}{l}\text { ((valor de estoque ini- } \\
\text { cial + valor de estoque } \\
\text { final / 2) × } 30 \text { / valor } \\
\text { total de consumo por } \\
\text { farmácia no mês) }\end{array}$ & Mensal \\
\hline
\end{tabular}




\begin{tabular}{|c|c|c|c|c|c|}
\hline \multirow{4}{*}{ Dispensação } & 4 & $\begin{array}{l}\text { Prescrições } \\
\text { médicas } \\
\text { atendidas in- } \\
\text { tegralmente }\end{array}$ & $\begin{array}{l}\text { Informa o per- } \\
\text { centual das pres- } \\
\text { crições médicas } \\
\text { atendidas integral- } \\
\text { mente por farmá- } \\
\text { cia por dia }\end{array}$ & $\begin{array}{l}\text { (№ total de pres- } \\
\text { crições médicas } \\
\text { atendidas integral- } \\
\text { mente / no total de } \\
\text { prescrições médicas } \\
\text { por farmácia por dia) } \\
\text { × } 100\end{array}$ & Diário \\
\hline & 5 & $\begin{array}{l}\text { Prescrições } \\
\text { de enferma- } \\
\text { gem atendi- } \\
\text { das integral- } \\
\quad \text { mente }\end{array}$ & $\begin{array}{l}\text { Informa o percen- } \\
\text { tual de prescrições } \\
\text { de enfermagem } \\
\text { atendidas integral- } \\
\text { mente por farmá- } \\
\text { cia por dia }\end{array}$ & $\begin{array}{l}\text { (№ total de prescri- } \\
\text { ções de enfermagem } \\
\text { atendidas integral- } \\
\text { mente / no total de } \\
\text { prescrições de enfer- } \\
\text { magem por farmácia } \\
\text { por dia) x } 100\end{array}$ & Diário \\
\hline & 6 & $\begin{array}{c}\text { Falta de me- } \\
\text { dicamento } \\
\text { ao paciente } \\
\text { internado }\end{array}$ & $\begin{array}{l}\text { Monitora a falta } \\
\text { de medicamentos } \\
\text { padronizados pres- } \\
\text { critos e não atendi- } \\
\text { dos no horário ao } \\
\text { paciente internado }\end{array}$ & $\begin{array}{l}\text { (№ de medicamentos } \\
\text { padronizados não } \\
\text { atendidos no horário } \\
\text { prescrito / no total de } \\
\text { medicamentos padro- } \\
\text { nizados solicitados) } \\
\text { × } 100\end{array}$ & Diário \\
\hline & 7 & $\begin{array}{c}\text { Erros na } \\
\text { dispensação } \\
\text { de medica- } \\
\text { mentos }\end{array}$ & $\begin{array}{l}\text { Informa a ocor- } \\
\text { rência de erros na } \\
\text { atividade de sepa- } \\
\text { ração/dispensação } \\
\text { de medicamentos } \\
\text { para atendimento } \\
\text { ao paciente } \\
\end{array}$ & $\begin{array}{l}\text { (№ medicamentos } \\
\text { dispensados com erro } \\
\text { registrados pela en- } \\
\text { fermagem / no total } \\
\text { de medicamentos } \\
\text { dispensados por far- } \\
\text { mácia por mês) x } 100\end{array}$ & Mensal \\
\hline Devolução & 8 & Estorno & $\begin{array}{l}\text { Informa o percen- } \\
\text { tual de estorno de } \\
\text { insumos não utili- } \\
\text { zados pelo pacien- } \\
\text { te em relação do } \\
\text { total de insumos } \\
\text { dispensados }\end{array}$ & $\begin{array}{l}\text { (№ total de devo- } \\
\text { luções / no total de } \\
\text { itens dispensados por } \\
\text { farmácia por mês) } \\
\text { x } 100\end{array}$ & Mensal \\
\hline $\begin{array}{l}\text { Reatendimen- } \\
\text { tos }\end{array}$ & 9 & $\begin{array}{l}\text { Reatendi- } \\
\text { mento de } \\
\text { medicamen- } \\
\text { tos }\end{array}$ & $\begin{array}{l}\text { Informa o per- } \\
\text { centual de rea- } \\
\text { tendimento de } \\
\text { medicamentos por } \\
\text { farmácia por dia }\end{array}$ & $\begin{array}{l}\text { (№ total de medica- } \\
\text { mentos reatendidos } \\
\text { / no total de medica- } \\
\text { mentos dispensados } \\
\text { por farmácia por dia) } \\
\times 100\end{array}$ & Diário \\
\hline
\end{tabular}

Fonte: Elaborado pelos autores.

O resultado do $\alpha$ calculado para a matriz de indicadores foi de 0,7616 . Já o coeficiente de alfa de Cronbach dos indicadores variou de 0,6307 a 0,7952. Os resultados individuais mostram que o indicador Satisfação do cliente apresentou coeficiente de alfa de Cronbach igual a 0,63, sendo considerado inferior ao valor mínimo aceitável, portanto sendo excluído da matriz de indicadores,

A Tabela 1 apresenta as metas estabelecidas aos indicadores e seus respectivos pesos propostos pelos autores. 
Tabela 1 - Metas e pesos estabelecidos para os indicadores da dimensão Gestão do Medicamento

\begin{tabular}{|c|c|c|}
\hline Nome do indicador & $\begin{array}{c}\text { Meta do indi- } \\
\text { cador }\end{array}$ & $\begin{array}{l}\text { Peso sugerido } \\
\text { pelos autores }\end{array}$ \\
\hline \multirow{3}{*}{ Acuracidade dos estoques } & $>95 \%$ & 1,5 \\
\hline & $75-95 \%$ & 1,0 \\
\hline & $55-75 \%$ & 0,5 \\
\hline \multirow{2}{*}{ Prescrições médicas atendidas integralmente } & $>95 \%$ & 1,25 \\
\hline & $75-95 \%$ & 0,75 \\
\hline \multirow{2}{*}{ Falta de medicamento ao paciente internado } & $0 \%$ & 1,25 \\
\hline & $>0 \%$ & 0,5 \\
\hline \multirow{2}{*}{ Atendimento do abastecimento } & $100 \%$ & 1,00 \\
\hline & $<100 \%$ & 0,5 \\
\hline \multirow{2}{*}{ Prescrições de enfermagem atendidas integralmente } & $>95 \%$ & 1,00 \\
\hline & $75-95 \%$ & 0,5 \\
\hline \multirow{2}{*}{ Erros na dispensação de medicamentos } & $0 \%$ & 1,00 \\
\hline & $>0 \%$ & 0,5 \\
\hline \multirow{2}{*}{ Dias de estoque } & $0-5$ dias & 1,00 \\
\hline & $>5$ dias & 0,5 \\
\hline \multirow{2}{*}{ Reatendimento de Medicamentos } & $0 \%$ & 1,00 \\
\hline & $>0 \%$ & 0,5 \\
\hline \multirow{2}{*}{ Estorno } & $0-10 \%$ & 1,00 \\
\hline & $>10 \%$ & 0,5 \\
\hline
\end{tabular}

Fonte: Elaborada pelos autores.

\section{DISCUSSÃO}

Este trabalho propôs as seguintes dimensões: Gestão da Assistência, Gestão de Recursos Humanos, Gestão do Medicamento, Gestão Financeira e Gestão da Clínica do Paciente. O fato de que a priorização por parte dos especialistas da dimensão a ser avaliada tenha resultado na Gestão do Medicamento mostra uma correlação com os achados na literatura, em que a Assistência Farmacêutica (AF) ainda é vista com enfoque na aquisição e distribuição de medicamentos. ${ }^{20-22}$

A gestão da qualidade na Farmácia Hospitalar não deve ser focada somente no produto de qualidade, adquirido ou manipulado, mas também nos serviços, tendo como objetivo garantir aos pacientes a melhor qualidade possível, reduzindo riscos e aumentando os casos de sucesso na terapêutica ${ }^{23} \mathrm{~A}$ qualidade é um conceito dinâmico, que muda seus referenciais ao longo do tempo. Conhecer a percepção de qualidade dos pacientes e profissionais permite melhor direcionar as estratégias e ações e propiciar uma tomada de decisão mais compatível com o contexto e objetivos da instituição de saúde. ${ }^{21,24}$

No setor da saúde, a busca por qualidade tem sido fortemente associada a programas de qualidade, os quais apresentam uma forte tendência em avaliar as condições dos hospitais, focando na estrutura, nos processos e nos resultados..$^{25}$ Essas dimensões foram amplamente utilizadas em diversos setores em busca de mensurar a qualidade dos seus produtos e serviços. Ao longo dos anos, no entanto, novos domínios veem sendo relatados, como os domínios organizacio- 
nal, operacional e de sustentabilidade. ${ }^{26}$ Da mesma forma, uma avaliação com a proposição de dimensões baseadas em processos observados começa a ser descrita em literatura. ${ }^{17,22,27}$ Nove indicadores foram propostos para a avaliação e monitoramento da dimensão da Gestão de Medicamento conforme metodologia apresentada.

O Indicador 1, acuracidade dos estoques, corresponde às diferenças encontradas entre as quantidades no estoque físico e as quantidades constantes no sistema, revelando-se um indicador de qualidade e confiabilidade dos dados. Detectar o problema que ocasionou a diferença pode ser oneroso, mas há uma necessidade de precisão dos estoques para a provisão adequada das demandas. ${ }^{28}$ Os percentuais de divergência aceitáveis variam conforme a empresa, mas que geralmente estão ligadas à representação financeira, facilidade de reposição e ao impacto de sua falta. ${ }^{28}$ Eles ainda mencionam que no mercado costuma-se tolerar divergências de até $1 \%$ para itens da curva A, $2 \%$ para itens da curva B e até $5 \%$ para itens da curva C. Para a matriz a meta proposta é de $95 \%$ de acurácia dos estoques, mas esta métrica pode ser adaptada de acordo com a realidade de cada instituição.

Os Indicadores 2, prescrições médicas atendidas integralmente, e 5, prescrições de enfermagem atendidas integralmente, relacionam-se com a capacidade de atendimento completo das necessidades dos pacientes e na execução do cuidado pela equipe de assistência. Esses indicadores auxiliam na medida do processo de dispensação. Avaliações relacionadas ao percentual de medicamentos prescritos e atendidos são relatadas em literatura com fortes evidências em atenção básica e são utilizados para monitorar a gestão dos serviços..$^{29,30}$ Para atendimento hospitalar, no entanto, não foram encontradas evidências. Entende-se que medir algo cujo atendimento deve ser integral evidencia falhas na qualidade do atendimento. No entendimento do grupo de especialistas, esta medida deve ser monitorada por prazo finito, na frequência proposta, com o objetivo de detectar falhas nos processos de dispensação e ajustes de sistema. A meta estabelecida foi de $95 \%$ para ambos os indicadores.

O Indicador 3, falta de medicamentos ao paciente internado, influencia diretamente na credibilidade da instituição prestadora da assistência, na garantia da qualidade do atendimento e na segurança dos pacientes atendidos pela instituição hospitalar. O controle eficaz do estoque influencia no gerenciamento desses medicamentos e evidencia a importância da adoção de processos de controle e ferramentas de gestão de estoques. ${ }^{31}$

O Indicador 6, erro de dispensação de medicamentos, é definido como a discrepância entre a ordem escrita na prescrição médica e o atendimento dessa ordem. No ambiente hospitalar, são erros cometidos por funcionários da farmácia (farmacêuticos, inclusive) quando realizam a dispensação de medicamentos para as unidades de internação. Muitos desses erros podem ser interceptados pelos profissionais de enfermagem, não atingindo os pacientes. A prescrição eletrônica elimina a dificuldade na leitura e no entendimento ocasionados pela letra ilegível do médico e possibilita que os erros de digitação sejam corrigidos no momento da elaboração da prescrição. ${ }^{32}$

Editora Unijuí - Revista Contexto \& Saúde - ISSN 2176-7114 - v. 21, n. 44, out./dez. 2021 
De acordo com outro estudo a taxa de erro de dispensação de 2015 a 2016 foi de 2,61\%, considerando o hospital de Pronto-Atendimento, Centro de Tratamento Intensivo (CTI) e Unidades de Internação. ${ }^{33} \mathrm{~A}$ meta estabelecida para o indicador foi definida como zero para acompanhamento da série histórica.

O indicador 7, dias de estoque, indica a quantidade em unidades de tempo (dias) em que o estoque médio será suficiente para cobrir a demanda média sem a necessidade de reposição. Leva em consideração o valor financeiro do estoque e o valor do Consumo Médio Mensal (CMM), calculando o número de dias cobertos, ${ }^{34} \mathrm{~A}$ meta estabelecida é muito variável, pois tem relação direta com a política de compra de cada instituição, modalidade de compra, entre outros fatores. A meta estabelecida relaciona-se à periodicidade de atendimento da farmácia pela Central de Abastecimento Farmacêutico (CAF) e valor de estoque avaliado, sendo limitada a cinco dias de estoque.

Para o Indicador 8, reatendimento de medicamentos, definido como atendimento em duplicidade mediante solicitação expressa da enfermagem, para este indicador não foram localizadas referências bibliográficas. Conceitualmente, esse indicador tem por objetivo monitorar o número de medicamentos dispensados acima da quantidade estabelecida pelo médico, no entanto solicitados pela equipe de enfermagem, mediante uma condição de excepcionalidade. Estão vinculados a esse processo dois fatores críticos, um quantitativo e outro qualitativo, o ressarcimento do medicamento e o monitoramento da justificativa para $\mathrm{o}$ atendimento dessa condição, respectivamente. $\mathrm{O}$ indicador proposto visa a contemplar a medida quantitativa, cuja meta foi estimada em zero.

Quando um medicamento não é administrado, o processo esperado é que ocorra a devolução dos medicamentos distribuídos às unidades para a farmácia hospitalar, processo representado pelo Indicador 9, denominado "estorno". Altos índices de devolução podem ter causas variadas, que vão desde um alto índice de prescrição de medicamentos para uso, se necessário, incluindo óbito e erros de aprazamento. ${ }^{32} \mathrm{O}$ excesso de medicamentos disponíveis nas unidades pode ser perigoso aos pacientes e aos profissionais de saúde. A meta de $8 \%$ foi baseada no histórico de hospitais de porte médio de aproximadamente 120 leitos. ${ }^{34}$ A meta estabelecida para este estudo para o estorno levou em consideração o histórico do serviço em torno de $10 \%$.

As metas devem ser estabelecidas utilizando-se um histórico, pesquisa de mercado, referenciais de comparação ou mesmo diretrizes institucionais. Entende-se que as metas estabelecidas aos indicadores propostos representam resultados ideais, no entanto faz-se necessário revisitar as medidas para que seja factíveis de serem alcançadas pois, caso contrário, podem tornar-se um fato de obrigatoriedade de uma demanda institucional. Além disso, a matriz de avaliação deve ser vinculada ao processo rotineiro da equipe pois, além de subsidiar a tomada de decisão, deve ser entendida como uma prática capaz de promover o aprendizado pessoal e institucional, melhoria contínua dos processos de gestão e, portanto, maior transparência e responsabilização dos membros da equipe. ${ }^{24}$

A matriz de avaliação e monitoramento da Farmácia Hospitalar proposta possui cinco dimensões: Gestão da Assistência, Gestão de Recursos Humanos, Gestão do Medicamento, Gestão Financeira e Gestão da Cínica do Paciente. A 
dimensão Gestão do Medicamento foi priorizada pelos especialistas e resultou num conjunto de nove indicadores. O resultado da priorização pelos especialistas evidenciou a tendência observada em literatura, em que a AF ainda é vista com enfoque na aquisição e distribuição de medicamentos e, sem esta etapa, pouco efetivas serão as demais.

A validação dos indicadores em termos de conteúdo e valoração permitiram atribuir um valor numérico como resultado da avaliação para a dimensão priorizada, atuando como uma ferramenta educativa, uma vez que a valoração permite identificar pontos fortes e fracos, contribuindo para a troca de experiências e resultados entre os serviços, ultrapassando apenas um caráter meramente fiscalizador dos indicadores. Dessa forma, cada fase de desenvolvimento da pesquisa contemplada no trabalho evidencia a capacidade de estruturação e delineamento de modelos com a participação ativa dos membros da equipe, aproximando os conceitos teóricos, dominados pelo meio acadêmico e sua aplicação dentro da problemática do campo prático. O farmacêutico é o profissional que tem sua formação fundamentada na articulação do conhecimento das áreas biológicas e exatas, atuando além da tradicional gestão de medicamentos, na criação de estratégias para promoção do uso racional de medicamentos.

A visão do profissional desta área tem buscado apresentar informações que possam fornecer dados para subsidiar a tomada de decisão, com o monitoramento por meio de indicadores entendido enquanto prática sendo capaz de promover o aprendizado pessoal e institucional e melhoria contínua dos processos de gestão. ${ }^{24}$ Muitos profissionais, no entanto, deparam-se com etapas do processo de avaliação sem o conhecimento necessário para compreensão e aplicação dos resultados.

O fato de apenas metade dos farmacêuticos convidados terem comparecido não compromete o resultado do método, visto que este total atende requisitos relativos à experiência e representatividade.

Não foram considerados os artigos não disponíveis em acesso livre, caracterizando-se como uma possível limitação metodológica da revisão bibliográfica. Além disso, os indicadores foram elaborados considerando uma realidade da Farmácia Hospitalar, podendo ser usados por outras instituições, mas recomenda-se a avaliação se refletem a realidade ou se as metas e os pesos adotados são adequados.

\section{CONSIDERAÇÕES FINAIS}

O estudo de avaliação é capaz de produzir informações para o julgamento da efetividade das ações. O número de artigos selecionados na busca bibliográfica mostra que são escassos os trabalhos que versam sobre o serviço de Farmácia Hospitalar (FH) e indicadores de performance.

Isso posto, o estudo apresentado contribuiu para evidenciar a capacidade de execução de métodos de consenso para a elaboração de indicadores aplicados à realidade de uma farmácia hospitalar. O instrumento desenvolvido para o acompanhamento da dimensão Gestão do Medicamento mostrou evidência de validade e uma boa confiabilidade. A nota máxima de dez pontos distribuída en-

Editora Unijuí - Revista Contexto \& Saúde - ISSN 2176-7114 - v. 21, n. 44, out./dez. 2021 
tre os nove indicadores permite a atribuição de um resultado, com concessão de valor, para a dimensão Gestão do Medicamento, permitindo assim a avaliação e o monitoramento das farmácias, contribuindo para melhorar a qualidade dos serviços.

\section{REFERÊNCIAS}

${ }^{1}$ Brasil. Portaria № 4.283, de 30 de dezembro de 2010. Aprova as diretrizes e estratégias para organização, fortalecimento e aprimoramento das ações e serviços de farmácia no âmbito dos hospitais. Diário Oficial da União. Brasília. 2010 dez. [Acesso em: 4 abr. 2018]. Disponível em: http://bvsms.saude.gov.br/bvs/saudelegis/gm/2010/ prt4283_30_12_2010.html

2 Sbrafh. Sociedade Brasileira de Farmácia Hospitalar. SBRAFH: dez anos de história. [Acesso em: 16 nov. 2017]. Disponível em: http://www.sbrafh.org.br/documentos/historico.pdf.

${ }^{3}$ Marin N, Consendey MAE, Luiza VL, Osorio-de-Castro CGC, Castro MS, Castilho SR, Noblat LACB. Por que diagnosticar a situação da Farmácia Hospitalar no Brasil? In: Osório-de-Castro CGS, Castilho SR. Diagnóstico da farmácia hospitalar no Brasil. Rio de Janeiro: Escola Nacional de Saúde Pública, Fundação Oswaldo Cruz; 2004. p. 17-33.

${ }^{4}$ Margarino-Torres R, Pepe VLE, Osorio-de-Castro CGS. Aspectos da avaliação de serviços na farmácia hospitalar brasileira. Rev Bras Farm. 2011;92(2):55-59.

${ }^{5}$ Donabedian A. The Quality of Medical Care. Science 200; 1978.

${ }^{6}$ Dias OV, Ramos LH, Costa SDM. Avaliação da qualidade dos serviços de saúde na perspectiva da satisfação dos usuários. Revista Pró-univer SUS. 2010 jul.;1(1):11-26.

${ }^{7}$ Bonato VL. Gestão de qualidade em saúde: melhorando a assistência ao cliente. O Mundo da Saúde. 2011 maio;35(5):319-331.

8 Lima TDM, Aguiar PM, Storpirtis S. Evaluation of quality indicator instruments for pharmaceutical care services: A systematic review and psychometric properties analysis. Res Social Adm Pharm. São Paulo. 2017 maio;14(5):405-412.

${ }_{9}^{9}$ Abuelsoud N. Pharmacy quality improvement project to enhance the medication management process in pediatric patients. Ir J Med Sci. 2018 jun.;188(2):591-600.

${ }^{10}$ Gomes ME, Barbosa E. A técnica de grupos focais para obtenção de dados qualitativos. Educativa. 1999 fev.;1(1):1-7.

${ }^{11}$ Cunha CM, Neto OPDA, Stackfleth R. Principais métodos de avaliação psicométrica da confiabilidade de instrumentos de medida. Rev. Aten. Saúde. 2016 set.;14(48):98-103.

${ }^{12}$ Alexandre NMC, Gallasch CH, Lima MHM, Rodrigues RCM. A confiabilidade no desenvolvimento e avaliação de instrumentos de medida na área da saúde. Rev. Eletr. Enferm. 2013 set.;15(3):802-809.

${ }^{13}$ Coluci MZO, Alexandre NMC, Milani D. Construção de instrumentos de medida na área da saúde. Ciênc. Saúde Coletiva. 2015 mar.; 20(3): 925-936.

${ }^{14}$ Souza ACD, Alexandre NMC, Guirardello EDB. Propriedades psicométricas na avaliação de instrumentos: avaliação da confiabilidade e da validade. Epidemiol. Serv. Saúde. 2017 set.;26(3):649-659.

15 Hora HRMD, Monteiro GTR, Arica J. Confiabilidade em questionários para qualidade: um estudo com o coeficiente Alfa de Cronbach. Produto \& Produção. 2010 jun.;11(2):85-103.

${ }^{16}$ Da Silva Medeiros RK, Ferreira Junior MA, De Souza Rego Pinto DP, Fortes Vitor A, Pereira Santos VE, Barichello E. Modelo de validação de conteúdo de Pasquali nas pesquisas em Enfermagem. Rev de Enferm. 2015 jan./fev.;4(4):127-135.

${ }^{17}$ Arkaravichien W, Wongpratat A, Lertsinudom S. Quality indicators to compare accredited independent pharmacies and accredited chain pharmacies in Thailand. Int J Clin Pharm. 2016 abr.;38(4):899-907.

${ }^{18}$ Trap B. Supervision, Performance Assessment, and Recognition Strategy (SPARS) - a multipronged intervention strategy for strengthening medicines management in Ugan- 
da: method presentation and facility performance at baseline. J Pharm Policy Pract. 2016 maio;9(21),1-15.

${ }^{19}$ Teichert M, Schoenmakers T, Kylstra N, Mosk B, Bouvy ML, Vaart FV, Smet PAGM, Wensing M. Quality indicators for pharmaceutical care: a comprehensive set with national scores for Dutch community pharmacies. Int J Clin Pharm. 2016 ago.;38(4):870-879.

20 Penaforte TR, Forster AC, Simões MJS. Evaluation of the performance of pharmacists in terms of providing health assistance at a university hospital. Clinical Science. 2007 jun.;62(5):567-572.

${ }^{21}$ Barreto JL, Guimarães MDCL. Avaliação da gestão descentralizada da Assistência Farmacêutica básica em municípios baianos. Cad. Saúde Pública. 2010 jun.;26(6):12071220.

22 Santana RS, Santos ADS, Menezes MS, Jesus EMSD, Silva WBD. Assistência farmacêutica de uma rede de hospitais públicos: proposta de utilização das diretrizes ministeriais para avaliação do serviço. Rev Bras Farm Hosp Serv Saúde. 2013 jan./mar.;4(1):29-34.

${ }^{23}$ Silva MJSD, Magarinos-Torres R, Oliveira MA, Osorio-de-Castro CGS. Avaliação dos serviços de farmácia dos hospitais estaduais do Rio de Janeiro, Brasil. Ciênc. Saúde Coletiva. 2013 out.;18(12):3605-3620.

${ }^{24}$ Santos JAD, Limberger JB. Indicadores de avaliação da assistência farmacêutica na acreditação hospitalar. Rev Adm Saúde. 2018 Jan.;18(70):1-17.

${ }_{25}$ Margarino-Torres R, Pepe VLE, Osorio-de-Castro CGS. Critérios e indicadores de resultados para a farmácia hospitalar brasileira utilizando o método Delfos. Cad Saúde Pública. 2007 ago.;23(8):1791-1802.

${ }^{26}$ Guimarães MCL, Santos SMC, Melo C, Sanches Filho. A avaliação da capacidade de gestão de organizações sociais: uma proposta metodológica em desenvolvimento. Cad Saúde Pública. 2004 nov./dez;20(6):1642-50.

${ }^{27}$ Costa LA, Santana GS, Pinto CR, Sampaio BC. Diagnóstico dos serviços de farmácia hospitalar da Secretaria de Saúde do Estado da Bahia. J Assist Farmac Farmacoecon. 2016 nov.;1(2):24-32.

${ }^{28}$ Ferranti E. Gestão de estoque de medicamentos utilizando classificação $A B C$ em um hospital público. Perspectiva Econômica. 2017 dez.;13(3):215-229.

${ }^{29}$ Melo DOD, Silva SRA, Castro LLC. Avaliação de indicadores de qualidade de prescrição de medicamentos em uma unidade de atenção primária com diferentes modelos de atenção. Epidemiol Serv Saúde. 2016 jun.;25(2):259-270.

${ }^{30}$ Mortari C, Henn RL, Maria V, Paniz V. Avaliação dos indicadores de prescrição e dispensação de medicamentos no município de Feliz/RS. Rev Bras Far. 2014 dez.;95(3):833854.

${ }^{31}$ Pereira LMV, Abramovicius AC, Ungari AQ, Oliveira HBD, Aragon DC, Costa AL, Forster AC. Descrição de prática para a gestão da farmácia hospitalar. Medicina. 2017 jul.;50(1):66-75.

${ }^{32}$ Limberger JB, Santos TS, Prediger KC, Ferrony D, Bertagnolli SMM. Análise do fluxo de distribuição e estorno de medicamentos em hospital filantrópico de Santa Maria, RS. Revista de Administração Hospitalar e Inovação em Saúde. 2013 maio;10(1):36-44.

${ }^{33}$ Oliveira ST, Farias PO, Drummond BM, Rodrigues LB, Reis PG, Souza LO, Oliveira LR, Miranda VF. Taxas de erro de prescrição e dispensação de um hospital público especializado em urgência e trauma. Rev Med Minas Gerais. 2018 dez.;28(5):61-68.

${ }^{34}$ Silva PL, Castilho SRD, Ferraz CVVG. Análise dos resultados da aplicação de práticas gerenciais na logística de estoque de uma farmácia hospitalar. Revista de Administração Hospitalar e Inovação em Saúde. 2017 out.;14(2):14-31. 